\title{
Fortification of dairy product Narine with polysaccharides from oyster mushroom
}

\author{
Ekaterina Antonceva ${ }^{1, *}$ and Mark Shamtsyan ${ }^{1}$ \\ ${ }^{1}$ Saint-Petersburg State Institute of Technology, 190013, Moskovsky pr., 26, Saint-Petersburg, Russia
}

\begin{abstract}
Mushrooms are rich in biologically active substances. Basidiomycete oyster mushroom or Pleurotus ostreatus is not only widely used in food, but also have radioprotection, preventive effect on recurrent respiratory tract infections, hypocholesterolic action, antitumor effect, strong immunostimulatory activity and other beneficial medical effects. It is considered that $\beta$-glucans play significant role in the biological activities of the oyster mushroom. The aim of this research was to study the effect of the addition of various $\beta$-glucan containing preparations obtained from the submerged biomass of $P$. ostreatus on the process of milk fermentation and properties of the finished product. Preparations of $\beta$-glucans obtained from submerged cultured $P$. ostreatus biomass were obtained using different extraction protocols. The content of $\beta$-glucans in preparations was determined using Assay Kit (Megazyme). Preparations were added to milk in different concentrations before the introduction of starter culture. A starter containing Lactobacillus acidophilus n.v. Er 317/402 strain «Narine» was used for fermentation. The titratable acidity was controlled during the fermentation. Physicochemical, structural-mechanical properties (water holding capacity) of the obtained samples were studied. The sensory evaluation of the products was evaluated. Results show that the addition of preparations increase the glycolytic activity. Structural-mechanical properties of yogurt samples fortified by $\beta$-glucan-containing preparations depend on the preparation and its concentration. Sensory evaluation showed that experimental samples differed not only from the control sample but also from each other.
\end{abstract}

\section{Introduction}

Diary products are among the most widely consumed foods. Enrichment of fermented milk products with various non-starchy polysaccharides, in particular, $\beta$-glucans and the creation of new functional products on their basis is a promising task, the solution of which can contribute to improving the health and quality of life of the population [1].

Prebiotics are partially or completely indigestible foods. They partially stimulate the growth and / or metabolism of one or more groups of microorganisms living in the large intestine, the normal composition of the intestinal microbiocenosis [2, 3]. Essentially, prebiotics feed microorganisms of the gastrointestinal tract. They are represented by

*Corresponding author:_p_m_@mail.ru 
disaccharides (disaccharides), oligosaccharides, polysaccharides, etc. The mechanism of action of all prebiotics is the same: without splitting under the influence of enzymes of the human small intestine, prebiotics enter the large intestine and are utilized mainly by bifidoand lactic acid bacteria $[4,5]$.

The main biological effects of prebiotics are:

- promote the growth of bifido- and lactic acid bacteria,

- stimulate intestinal peristalsis,

- increase calcium assimilation,

- exert hypocholesterolemic effect,

- reduce the risk of developing tumors [6].

Fermented milk product "Narine" is acidophilic milk fermented with culture Lactobacillus acidophilus n.v. Er 317/402. This product has a high antagonistic activity against opportunistic intestinal microflora. Besides, other therapeutic properties, probiotics also have antihypertensive and antidiabetic properties by modulating lipid profile and insulin resistance [7]. Lactobacillus acidophilus n.v. Er 317/402 strain «Narine» normalizes the intestinal microflora, restores the anaerobic flora (lactobacilli and bifidobacteria) in a short time, increases the activity of normal Escherichia coli and the antioxidant potential of the body, helps to reduce cholesterol levels $[8,9]$.

B-glucans are known to have a wide range of different biological actions. Their inclusion in the composition of food products and the study of the properties of finished products is promising. Scientists in many countries include $\beta$-glucans obtained from oats, barley, mushrooms, algae and other sources in functional foods to give them additional beneficial properties for humans [10]. In addition to health and nutritional benefits, $\beta$ glucans also have various suitable functional properties such as thickening, stabilizing, emulsifying and gelling. These properties determine the suitability of $\beta$-glucans for inclusion in soups, sauces, beverages, and other foods [11]. Glucans can find use in the production of cheese, low-fat ice cream, yoghurts, and can also be combined with other soluble fiber in low-fat dairy products and low-fat curd cheeses to improve gelation and rheology [11].

The most biologically active form of $\beta$-glucans is $\beta-1,3 / 1,6$-glucans, in the molecules of which the main chain is a polymer of D-glucose linked by $\beta-1,3$-glycosidic bonds, which also have $\beta$ branching -1,6-glycosidic bonds, found mainly in fungi and yeast [12]. Extracts containing fungal $\beta$-glucans are one of the most studied soluble dietary fiber, and are known for their beneficial effects on human health, such as reducing the glycemic response and serum cholesterol levels [13], as well as having antitumor activity and other beneficial effects [14].

Polysaccharides obtained from Pleurotus ostreatus possess a wide range of biological activity in vivo and in vitro as radioprotection, preventive effect on recurrent respiratory tract infections and others [15-17]. Thus, a significant reduction of cholesterol levels in mice was observed in serum $(31-46 \%)$ and liver $(25-30 \%)$ at a dose of $5 \%$ of oyster mushroom for all periods $(8,16,28$, and 52 weeks) was shown [18].

From $P$. ostreatus mycelium were extracted polysaccharides and an antitumor component, known as $P$. ostreatus mycelium polysaccharides 2 (POMP2). Results of the MTT assay indicated that POMP2 had a marked inhibitory effect on the BGC-823 human gastric cancer cell line. Furthermore, in vivo tests of mice engrafted with BGC-823 cancer cells demonstrated that both tumor weight and volume were markedly reduced following two weeks of treatment with POMP2 [19].

The $P$. ostreatus displayed strong immunostimulatory activity [20]. According to Sun and Liu tests in vitro showed water-soluble polysaccharide from the fruiting body of $P$. ostreatus is capable of enhancing concanavalin A or lipopolysaccharide-induced lymphocyte proliferation, which suggested that POP could be a potential 
immunostimulating agent for use in functional foods or medicine against both pathogens and cancer [21].

It is believed that polysaccharides isolated from P. ostreatus can be used to develop new functional products [10]. When incorporated into products, such substances can provide additional prophylactic and functional properties, such as immunostimulant, hypoglycaemic, anti-radical, antitumor, anti-inflammatory and others [22-24].

\section{Materials and methods}

\subsection{Preparations}

The process of culturing of $P$. ostreatus and isolating various preparations from dry crushed submerged biomass was described in detail by Antontceva et al. [25].

The following $\beta$-glucan contained preparations were obtained:

- water-insoluble preparation P1, which was collected after double processing by $80 \%$ ethanol;

- water-soluble preparation P2, collected after extraction of preparation P1 in a boiling water bath and then concentrated by evaporation and precipitated with $96 \%$ ethanol solution;

- and water-insoluble preparation P3, taken as the solid residue remaining after all extractions.

The content of $\beta$-glucans in preparations was determined using the $\beta$-glucan Assay Kit (Yeast \& Mushroom) (Megazyme, USA) [26].

\subsection{Preparation of fermented milks samples}

As a base for dairy product was used high-temperature (UHT) treated milk (Valio, "Galactica" plant) with a fat content of $0.05 \%$. For the fermentation of milk was used the following lyophilized starter culture for a fermented acidophilus milk - probiotic preparation "Narine" containing the Lactobacillus acidophilus n.v. Er 317/402 strain.

The fermented milk product "Narine" was grown on milk in flasks at a temperature of $40{ }^{\circ} \mathrm{C}$ until complete coagulation. One of the three preparations of a certain mass was initially added into sterile glasses, and then they were filled with milk up to a volume of $150 \mathrm{ml}$. At the end, the inoculum was added ( $5 \%$ of the sample volume).

For all samples the following dosages of the preparations were taken (Table 1):

Table 1. Analysed samples.

\begin{tabular}{|l|c|c|}
\hline \multicolumn{1}{|c|}{ Sampels } & $\begin{array}{c}\text { Type of } \\
\text { preparation }\end{array}$ & $\begin{array}{c}\text { Concentration of } \\
\text { preparation, \% }\end{array}$ \\
\hline Control & - & - \\
\hline Experimental P1 $-0.1 \%$ & $\mathrm{P} 1$ & 0.1 \\
\hline Experimental P1 $-0.25 \%$ & $\mathrm{P} 1$ & 0.25 \\
\hline Experimental P2-0.1\% & $\mathrm{P} 2$ & 0.1 \\
\hline Experimental P2 $-0.25 \%$ & $\mathrm{P} 2$ & 0.25 \\
\hline Experimental P3-0.1\% & $\mathrm{P} 3$ & 0.1 \\
\hline Experimental P3 $-0.25 \%$ & $\mathrm{P} 3$ & 0.25 \\
\hline
\end{tabular}

Then the samples were thermostated during 8 hours at the temperature recommended by the starter manufacturer, with periodic stirring and sampling to determine the titratable acidity. 


\subsection{Characteristics of dairy products}

\subsubsection{Determination of titratable acidity}

Analysis of titratable acidity was carried out according to the indicator method described in GOST (Russian State Standard) 3624-92 "Milk and dairy products. Titrimetric methods for determination of acidity".

\subsubsection{Determination of water-holding capacity (WHC)}

Water-holding capacity (WHC) of fermented milk were studied according to the procedure described in Sodini [26]. Samples of fermented milk (about $20 \mathrm{ml}$ ) (Y) after cooling to $4{ }^{\circ} \mathrm{C}$ and 24 hours of storage were centrifuged for 10 minutes at $4000 \mathrm{~min}^{-1}$ at $20^{\circ} \mathrm{C}$. The released serum (W) was removed and weighed. The WHC of fermented milk is calculated by the formula (1):

$$
W H C=(Y-W) / Y \times 100 \%
$$

\subsubsection{Sensory evaluation}

Samples organoleptic were evaluated according to the GOST (Russian standard) 22935-32011 "Milk and dairy products. Organoleptic analysis" and evaluate in points from 1 to 5 on the following scale (Table 2).

Table 2. Scale of organoleptic properties differentiation.

\begin{tabular}{|c|l|}
\hline Point & \multicolumn{1}{c|}{ Description } \\
\hline 5 & Extremely acceptable - no deviation from the previously established requirements \\
\hline 4 & Very acceptable - minimum deviation from the previously established requirements \\
\hline 3 & Acceptable - noticeable deviation from the previously established requirements \\
\hline 2 & Barely acceptable - significant deviation from the previously established requirements \\
\hline 1 & $\begin{array}{l}\text { Unacceptable - grossly significant deviation from the previously established } \\
\text { requirements }\end{array}$ \\
\hline
\end{tabular}

Sensory evaluation was performed by 16 volunteers in the age 20-60 using the scale above to judge the degree of compliance to the following quality requirements:

- appearance and consistency: must be homogeneous, fairly viscous, possibly with undissolved particles belonging to the added substances;

- taste and odour: clean, sour-milk, no off-flavours;

- colour: milky; either white or characteristic of the added substances.

Participation was free for anyone who wished to take part and had mainly students and staff of the Department of Technology of Microbiological Synthesis.

All experiments were carried out in three replicas (besides the hedonistic appraisal described below), with statistical processing of data, the level of confidence was taken as 0.95, and the Office Excel program was used.

\section{Results and discussion}

\subsection{Content of $\beta$-glucans in preparations}

The content of $\alpha$-glucans and total glucans were measured. The content of $\beta$-glucans was calculated. All the date are demonstrated in the Table 3. 
Table 3. Content of glucans in preparations.

\begin{tabular}{|c|c|c|c|}
\hline Sample & $\begin{array}{c}\text { Total } \\
\text { glucans, \% }\end{array}$ & $\begin{array}{c}\alpha \text {-glucans, } \\
\%\end{array}$ & $\begin{array}{c}\beta \text {-glucans, } \\
\%\end{array}$ \\
\hline Preparation P1 & $36,0 \pm 0,2$ & $3,0 \pm 0,2$ & $33,0 \pm 0,1$ \\
\hline Preparation P2 & $31,5 \pm 0,4$ & $7,6 \pm 0,2$ & $23,9 \pm 0,2$ \\
\hline Preparation P3 & $43,5 \pm 0,3$ & $2,3 \pm 0,1$ & $41,2 \pm 0,2$ \\
\hline
\end{tabular}

\subsection{Dynamics of titratable acidity}

The influence of the preparation from P. ostreatus on the milk fermentation process with starter culture L. acidophilus n.v. Er 317/402 "Narine" was studied. Dairy product fermented acidophilous milk was obtained.

During fermentation, the titratable acidity of the samples was determined every 2 hours (Table 4).

Table 4. Dynamics of changes in titratable acidity of different samples.

\begin{tabular}{|l|c|c|c|c|c|}
\hline \multirow{2}{*}{ Sample } & \multicolumn{5}{|c|}{ Titrable acidity, ${ }^{\circ} \mathrm{T}$} \\
\cline { 2 - 6 } & \multicolumn{5}{|c|}{ Duration, $\mathrm{h}$} \\
\cline { 2 - 6 } & 0 & 2 & 4 & 6 & 8 \\
\hline Control & $20 \pm 1$ & $35 \pm 2$ & $60 \pm 1$ & $98 \pm 2$ & $132 \pm 2$ \\
\hline P1 - 0.1\% & $20 \pm 1$ & $36 \pm 1$ & $60 \pm 2$ & $106 \pm 2$ & $138 \pm 1$ \\
\hline P1- $0.25 \%$ & $20 \pm 1$ & $35 \pm 2$ & $72 \pm 2$ & $109 \pm 3$ & $141 \pm 1$ \\
\hline P2-0.1\% & $21 \pm 1$ & $39 \pm 1$ & $81 \pm 2$ & $116 \pm 2$ & $166 \pm 3$ \\
\hline P2-0.25\% & $21 \pm 1$ & $36 \pm 2$ & $76 \pm 2$ & $114 \pm 3$ & $160 \pm 2$ \\
\hline P3-0.1\% & $20 \pm 1$ & $36 \pm 1$ & $64 \pm 2$ & $112 \pm 1$ & $148 \pm 1$ \\
\hline P3-0.25\% & $21 \pm 2$ & $36 \pm 2$ & $67 \pm 2$ & $112 \pm 3$ & $152 \pm 2$ \\
\hline
\end{tabular}

The addition of the preparations into milk considerably increased the glycolytic activity of starter cultures (Table 4).

Level of titratable acidity after 4 hours from the beginning of fermentation for samples (which contain preparation P2) was higher then the level of titratable acidity for control samples. In 8 hours of fermentation, it is observed that an addition of the preparations $\mathrm{P} 1$, P2 and P3 into milk before the fermentation did lead to a significant increase of the glycolytic activity of the culture. It may indicate the prebiotic effect of used functional additives.

\subsection{Water-holding capacity (WHC)}

One of the important structural-mechanical parameters which determine the texture and stability of a dairy product is water-holding capacity. Therefore, for all obtained product samples, the WHC was determined. The results of these studies for WHC of the clot are shown in Figure 1.

The obtained data show that the addition of all the preparations to the milk fermented with "Narine" containing the L. acidophilus culture causes an increase in the WHC of the milk clot. The preparations $\mathrm{P} 1, \mathrm{P} 2$ and $\mathrm{P} 3$ added at a concentration of $0.1 \%$ causes an increase in WHC by 13,12 , and $16 \%$, respectively, compared to the control sample. Preparations P1 and P2, added at concentrations of $0.25 \%$, caused an increase in WHC by 43 and 39\%, respectively, compared with the control sample. The preparation P3, added at a concentration of $0.25 \%$, causes an increase in WHC by $21 \%$, respectively, compared to the control sample. 
There was a correlation between WHC and acidity of fermented milks samples with preparations P1 and P3 which contained waterinsoluble fraction. The increase of acidity during fermentation strengthened the curd stability because of the increase in water-binding capacity of proteins [27]. Preparation P2 is completely water-soluble and not demonstrated this correlation.

Thus, the best results were shown by preparations P1 and P2 at a concentration of $0.25 \%$. This may be due to the presence of water-soluble $\beta$-glucans in both preparations, which can improve the texture of dairy products.

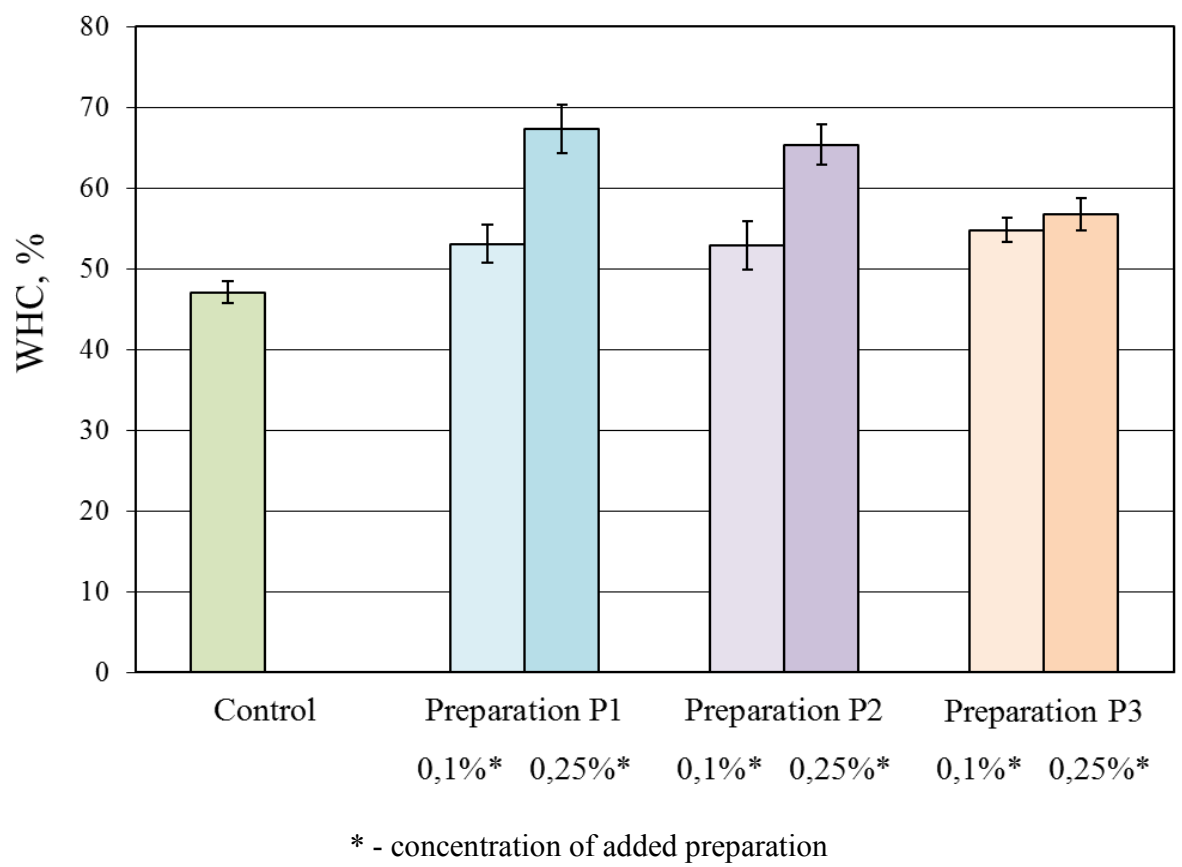

Fig. 1. The effect of addition of the preparations P1, P2 and P3 on the samples' water holding capacity $(\mathrm{p}<0.05)$.

\subsection{Sensory evaluation}

The organoleptic characteristics, such as taste, smell, color and consistency were analyzed. Evaluation of the sample in scores for samples of dairy products with and without preparations are shown in Table 5.

Table 5. Sensory evaluation of different samples.

\begin{tabular}{|l|c|c|c|c|c|}
\hline \multirow{2}{*}{ Sample } & \multicolumn{5}{|c|}{ Evaluation of the sample, scores } \\
\cline { 2 - 6 } & Taste & Odour & Colour & Appearance & Total \\
\hline Control & 5.0 & 5.0 & 5.0 & 5.0 & 20.0 \\
\hline P1 $-0.1 \%$ & 4.8 & 5.0 & 3.8 & 4.8 & 18.4 \\
\hline P1-0.25\% & 4.9 & 5.0 & 3.7 & 4.8 & 18.3 \\
\hline P2-0.1\% & 5.0 & 5.0 & 4.8 & 5.0 & 19.8 \\
\hline P2-0.25\% & 4.5 & 4.9 & 3.6 & 4.7 & 17.8 \\
\hline P3-0.1\% & 4.6 & 4.9 & 3.6 & 4.7 & 17.8 \\
\hline P3-0.25\% & 4.7 & 4.8 & 3.5 & 4.8 & 17.8 \\
\hline
\end{tabular}


The highest score was shown by the sample with the preparation P2 at a concentration of $0.1 \%$. It was chosen as the basis for further formulation improvement.

\section{Conclusion}

The present study demonstrates the possibility of using a $\beta$-glucan-containing preparation from the biomass of a culture $P$. ostreatus in a fermented milk product based on Narine's starter culture containing lactic acid bacteria L. acidophilus.

The addition of $P$. ostreatus preparations to milk increased the glycolytic activity of L. acidophilus culture. The titratable acidity of milk fermented with L.acidophilus increased as a result of the use of each of the preparations, the highest results were shown by the preparation $\mathrm{P} 2$, the titratable acidity was $23 \%$ higher than the control sample.

The introduction of drugs into milk before fermentation increased the WHC of the clots. Perhaps this is due to an increase in the content of dry substances leading to the strengthening of the structure of the fermented milk product.

The organoleptic evaluation of the obtained samples showed acceptable results. Some of the experimental samples were very similar in taste, colour and odour to the control sample, and the consistency was even better and the clot structure was more stable.

The addition of polysaccharide preparations has a positive effect on the lactic acid fermentation process carried out by L. acidophilus. The addition of polysaccharide preparations before the dairy fermentation stage can shorten the fermentation time and improve the physicochemical properties of fermented milk products. The results obtained suggest the possibility of using $\beta$-glucans of mushroom preparations to create functional food products based on fermented milk products.

\section{References}

1. P. Russo, P. López, V. Capozzi, P.F. De Palencia, M.T. Dueñas, G. Spano, D. Fiocco, International Journal of Molecular Sciences 13(5), 6026-6039 (2012) DOI: $10.3390 /$ ijms 13056026

2. G. Macori, P.D. Cotter, Current opinion in biotechnology 49, 172-178 (2018) DOI: 10.1016/j.copbio.2017.09.002

3. Y. Uyeno, S. Shigemori, T. Shimosato, Microbes and environments 30(2), 126-132 (2015) DOI: 10.1264/jsme2.ME14176

4. E.M. Quigley, Pharmacological research 61(3), 213-218 (2010) DOI: 10.1016/j.phrs.2010.01.004

5. C. Kunz, M. Rodriguez-Palmero, B. Koletzko, R. Jensen, Clinics in perinatology 26(2), 307-333 (1999) DOI: 10.1016/S0095-5108(18)30055-1

6. D. Davani-Davari, M. Negahdaripour, I. Karimzadeh, M. Seifan, M. Mohkam, S.J. Masoumi, A. Berenjian, Y. Ghasemi, Foods 8(3), 92(1-27) (2019) DOI: 10.3390/foods 8030092

7. H.S. Lye, C.Y. Kuan, J.A. Ewe, W.Y. Fung, M.T. Liong, International journal of molecular sciences 10(9), 3755-3775 (2009) DOI: 10.3390/ijms10093755

8. H. Charchoghlyan, J.E. Bae, H. Kwon, M. Kim, Biotechnology and Bioprocess Engineering 22(3), 327-338 (2017) DOI: 10.1007/s12257-017-0065-8

9. A. Pepoyan, M. Balayan, A. Manvelyan, L. Galstyan, S. Pepoyan, S. Petrosyan, V. Tsaturyan, S. Kamiya, T. Torok, M. Chikindas, Frontiers in immunology 9, 1426 (2018) DOI: 10.3389/fimmu.2018.01426 
10. I. Giavasis, Current opinion in biotechnology 26, 162-173 (2014) DOI: 10.1016/j.copbio.2014.01.010

11. A. Ahmad, F.M. Anjum, T. Zahoor, H. Nawaz, S.M.R. Dilshad, Critical reviews in food science and nutrition 52(3), 201-212 (2012) DOI: 10.1080/10408398.2010.499806

12. N. Dalonso, G.H. Goldman, R.M.M. Gern, Applied microbiology and biotechnology 99(19), 7893-7906 (2015) DOI: 10.1007/ s00253-015-6849-x

13. K.J. Shelat, F. Vilaplana, T.M. Nicholson, M.J. Gidley, R.G. Gilbert, Carbohydrate Polymers 86(4), 1732-1738 (2011) DOI: 10.1016/j.carbpol.2011.07.004

14. J.M. Facchini, E.P. Alves, C. Aguilera, R.M.M. Gern, M.L.L. Silveira, E. Wisbeck, S.A. Furlan, International journal of biological macromolecules 68, $72-77$ (2014) DOI: 10.1016/j.ijbiomac.2014.04.033

15. G. Llauradó, H.J. Morris, V. Tamayo, Y. Lebeque, Y. Beltrán, J. Marcos, R.C. Bermudez, Natural product research 29(16), 1557-1561 (2015) DOI: $10.1080 / 14786419.2014 .983918$

16. J. Pasnik, A. Ślemp, A. Cywinska-Bernas, K. Zeman, M. Jesenak, Current Pediatric Research 21(1), 99-104 (2017)

17. W. Radzki, M. Ziaja-Sołtys, J. Nowak, J. Rzymowska, J. Topolska, A. Sławińska, M. Michalak-Majewska, M. Zalewska-Korona, A. Kuczumow, LWT-Food Science and Technology 66, 27-33 (2016) DOI: 10.1016/j.lwt.2015.10.016

18. P. Bobek, Š. Galbavý, Food/Nahrung 43(5), 339-342 (1999) DOI: 10.1016/S08999007(97)00471-1

19. X.Y. Cao, J.L. Liu, W. Yang, X. Hou, Q.J. Li, Molecular medicine reports 12(2), 23832389 (2015) DOI: 10.3892/mmr.2015.3648

20. G.S. Deo, J. Khatra, S. Buttar, W.M. Li, L.E. Tackaberry, H.B. Massicotte, K.N. Egger, K. Reimer, C.H. Lee, International journal of medicinal mushrooms 21(07), 629-643 (2019) DOI: 10.1615/IntJMedMushrooms.2019031193

21. Y. Sun, J. Liu, Bioresource Technology 100(2), 983-986 (2009) DOI: 10.1016/j.biortech.2008.06.036

22. M.A. Khan, M. Tania, Food Reviews International 28(3), 313-329 (2012) DOI: $10.1080 / 87559129.2011 .637267$

23. M. Shamtsyan, Potential to develop functional food products from mushroom bioactive compounds, Journal of Hygienic Engineering and Design 15, $51-59$ (2016)

24. M. Shamtsyan, E. Antontceva, A. Panchenko, N. Petrishchev, Hyperlipidemic and hypocholesterolic action of submerge cultured mushrooms, Journal of Hygienic Engineering and Design 7, 96-99 (2014)

25. E. Antontceva, S. Sorokin, M. Shamtsyan, L. Krasnikova, Journal of Hygienic Engineering and Design 22, 47-52 (2018)

26. B.V. McCleary, A. Draga, Journal of AOAC International 99(2), 364-373 (2016) DOI: 10.5740/jaoacint.15-0289

27. I. Sodini, A. Lucas, M.N. Oliveira, F. Remeuf, G. Corrieu, Journal of Dairy Science 85(10), 2479-2488 (2002) DOI: $10.3168 / j d s . S 0022-0302(02) 74330-0$

28. D. Sert, E. Mercan, E. Dertli, LWT 78, 70-76 (2017) DOI: 10.1016/j.lwt.2016.12.023 